\title{
MAROC: Multi-Anode ReadOut Chip for MaPMTs
}

\author{
P. Barrillon, S. Blin, M. Bouchel, T. Caceres, C. de La Taille, member IEEE, G. Martin, P. Puzo, N. Seguin-Moreau \\ Laboratoire de l'Accélérateur Linéaire, IN2P3-CNRS, Université Paris Sud 11
}

For the ATLAS luminometer, made of Roman pots, a complete readout ASIC has been designed in $0.35 \mathrm{SiGe}$ technology. It is used to readout 64 channels multi anode photomultipliers and supplies 64 trigger outputs and a multiplexed charge. Since its delivery in November 2005, the MAROC chip has been tested at LAL. Despite a substrate coupling effect which affects the performance when all channels are used in high gain, the chip has shown nice global behavior and it has been used during beam tests at CERN in October 2006.

\section{INTRODUCTION}

MAROC is the readout chip designed for the ATLAS luminometer made of Roman pots [1]. This ASIC is an evolution of the OPERA_ROC ASIC [2] developed and installed on the OPERA experiment to auto-trigger and readout 64 channels Hamamatsu multi anode photomultiplier tubes (MAPMTs) [3]. Fig. 1 represents the layout of the first version of MAROC.

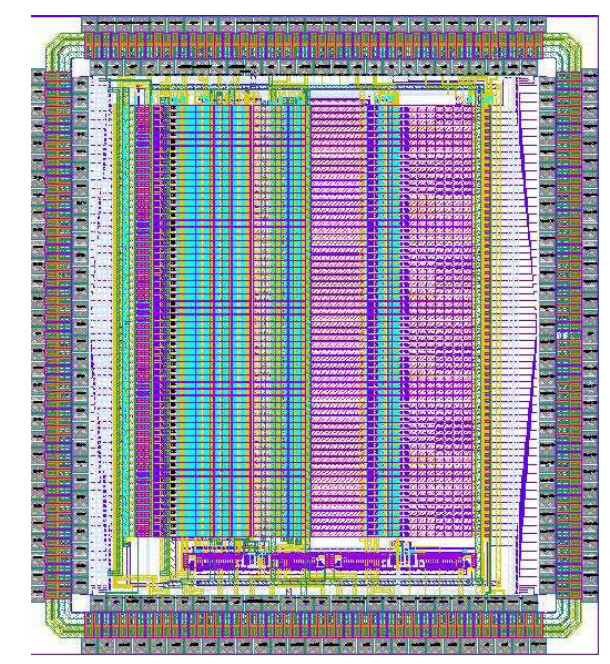

Fig. 1. Layout of the first version of MAROC.

In order to check the functionalities and performances of MAROC, laboratory tests have been performed thanks to a test board developed at LAL.

This paper will successively describe the ATLAS luminometer and the main features and requirements of the chip itself (section II), expose the results of the tests carried out at LAL (section III) and present the new version of the chip developed taking into account those results (section IV).

\section{DESCRIPTION}

\section{A. ATLAS Luminometer}

As previously said, the ATLAS luminometer is made of roman pots [1]. They consist in $0.5 \mathrm{~mm}^{2}$ scintillating fibers arranged in planes in the $\mathrm{U}$ and $\mathrm{V}$ directions. One roman pot is composed by 10 planes of 64 fibers in $\mathrm{U}$ and $\mathrm{V}$ as showed by fig. 2. Eight roman pots will be used with ATLAS detector and will be located at $240 \mathrm{~m}$ from the interaction point.

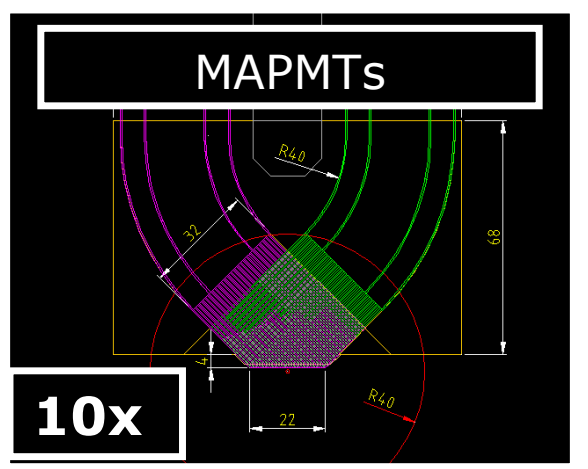

Fig. 2. Schematic of the scintillating fibers arranged for a roman pot.

The light produced by the scintillating fibers is captured by 64 channels multi-anode photomultiplier tubes $\mathrm{H} 7546$ from Hamamatsu. They will run at a high voltage of 800 to $950 \mathrm{~V}$ corresponding to a gain of $3.10^{5}$ to $2.10^{6}$. It is important to notice that there is a non uniformity of $1: 3$ between the channels of a single PMT.

Finally the PMTs are readout by MAROC. 200 chips will be needed for the final detector. They will be produced in 2007.

A complete review of the roman pots status is given in [4].

\section{B. The Multi-Anode Readout Chip}

\section{1) Main features}

The MAROC has been designed using the AMS SiGe $0.35 \mu \mathrm{m}$ technology. The package used is CQFP240. The area of the chip is $12 \mathrm{~mm}^{2}$. It has been submitted in June 2005 and received in November 2005.

Fig. 3. represents the bloc diagram of MAROC with its main features. The chip has 64 "super common base" inputs, 64 trigger outputs and a multiplexed charge output. Each channel is made of a variable gain preamplifier with 
low input tunable impedance (50-100 $\Omega$ ), a low offset and a low bias current $(20 \mu \mathrm{A})$ in order to minimize the cross talk. This variable gain allows compensating for the PM gain dispersion up to a factor 4 to an accuracy of $6 \%$ with 6 bits. The amplified current feeds then two paths:

- A slow shaper path which consists in a CRRC ${ }^{2}$ shaper and a Sample and Hold Widlar differential buffer. This S\&H block stores the charge in a $2 \mathrm{pF}$ capacitor and deliver a multiplexed charge measurement with a $5 \mathrm{MHz}$ readout speed.

- A fast (15 ns) shaper path made of a CRRC bipolar shaper followed by a discriminator. The threshold is set by an internal 10 bit DAC composed by a 4 bits thermometer DAC allowing coarse tuning $(200 \mathrm{mV}$ per step) and a 7 bits mirror DAC used for fine tuning (3 $\mathrm{mV}$ per step). A trigger output is produced.

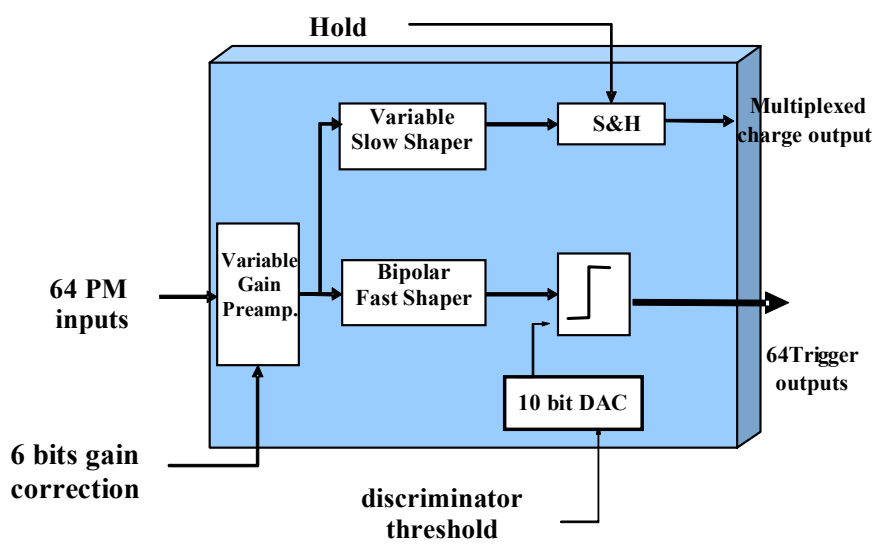

Fig. 3. Bloc diagram of the MAROC ASIC.

Fig. 4. gives a detailed view of the one channel circuit with the different parts described previously.

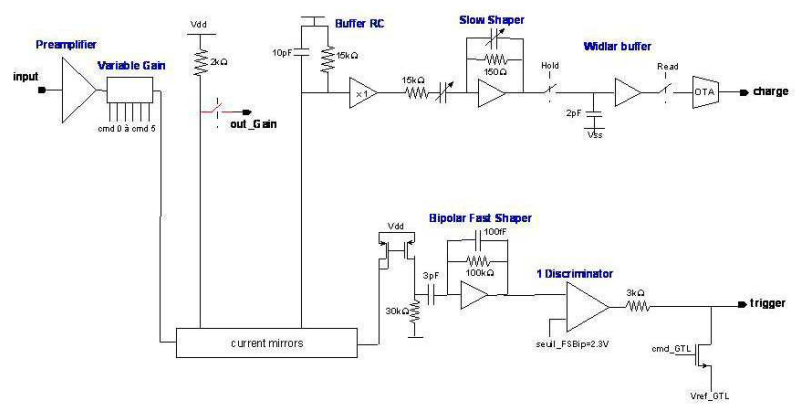

Fig. 4. One channel schematic.

\section{2) Requirements}

The main requirements concerning the MAROC are the following:

-A 0-4 variable gain preamplifier in order to correct for the PM non uniformity.
- The trigger efficiency must reach $100 \%$ for a signal larger than $1 / 3$ of photoelectron (pe), which corresponds to a charge of $50 \mathrm{fC}$ for a $\mathrm{PM}$ functioning at gain $10^{6}(900 \mathrm{~V})$.

-A charge measurement up to 30 photoelectrons with a linearity of $2 \%$ or better

- A cross talk of less than $1 \%$

-A noise of $2 \mathrm{fC}$ or better

In order to verify these requirements, many tests have been performed at laboratory. They are presented as well as the results in the next section.

\section{PERFORMANCES}

\section{A. Test set-up}

A test board has been developed at LAL in order to perform the characterization tests needed to verify the performances of MAROC. Fig. 5 represents a top view of this board with the chip mounted directly on board, the control FPGA (Altera), the USB and GPIB ports and the 64 channels PM socket are visible. This board was controlled through LabVIEW software [5] via the GPIB port.

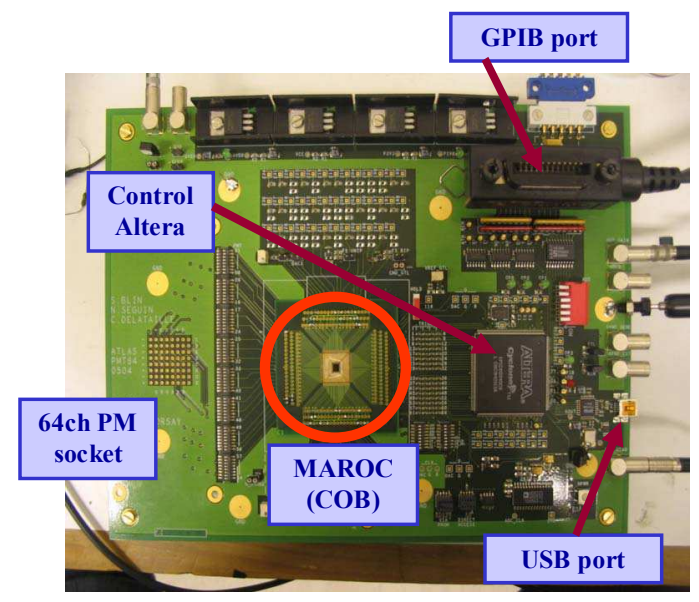

Fig. 5. Picture of the test board used for characterization tests.

In addition to the test board and the control PC, other equipments were used: a pulser to provide the input signal, a voltmeter and an oscilloscope in order to visualize the charge or trigger outputs.

The data analysis was performed mainly with Igor [6] and paw [7] software.

\section{B. Substrate coupling effect}

The first measurements made with this set-up were done with a chip mounted on a socket. They showed $100 \mathrm{MHz}$ oscillations when all channels were set at high gain $(\geq 1)$. Similar behavior was also seen in simulation.

After investigations, the reason of this effect was found to be the large inductance $(30 \mathrm{nH})$ between the ground of the chip and the test board ground and the existence of a substrate 
resistance $(\sim 2 \Omega)$ coupling the mirror output to the preamplifier ground return. This is symbolized by fig. 6 , where $\mathrm{L}_{\text {bonding, }} \mathrm{L}_{\text {package }}$ and $\mathrm{L}_{\text {socket }}$ are different contributions to the ground inductance coming from the use of the socket and $\mathrm{R}$ is the substrate resistance.

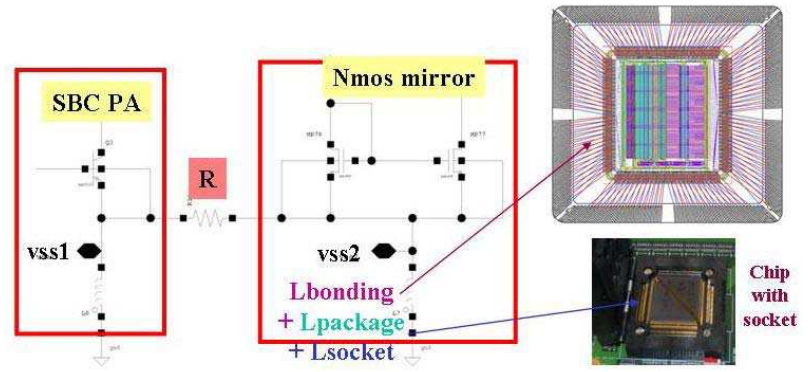

Fig. 6. Substrate resistance schematic and picture of a chip mounted on a socket.

It was decided to use the "chip on board" technology which has the clear advantage to remove the package and allow having the ground plan directly under the chip, reducing this way the inductance.

The rest of this section presents results obtained with a chip mounted on board.

\section{Pedestals}

Fig. 7 gives the pedestal distributions, for the 64 channels of a single chip, for the slow shaper (left) and fast shaper (right). In both case a small dispersion of 3-4 $\mathrm{mV}$ rms was obtained.
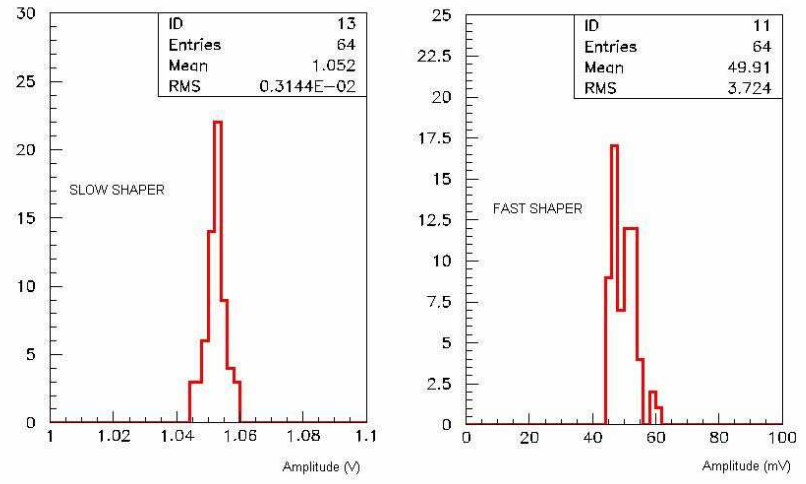

Fig. 7. Pedestal distributions for the slow and fast shapers.

\section{Charge output}

Waveforms were recorded with a fixed injection charge of 1 $\mathrm{pC}$ and for variable preamplifier gains as one can see on the fig. 8 which represents the amplitude as a function of the time for the 64 different gains. The waveforms with gains 1, 2 and 4 are highlighted. The amplitude ratios between the waveforms correspond approximately to this number. At gain
$\mathrm{G}=1$ the amplitude is $150 \mathrm{mV} / \mathrm{pC}$ which corresponds to 24 $\mathrm{mV} /$ pe for a MAPMT gain of $10^{6}$.

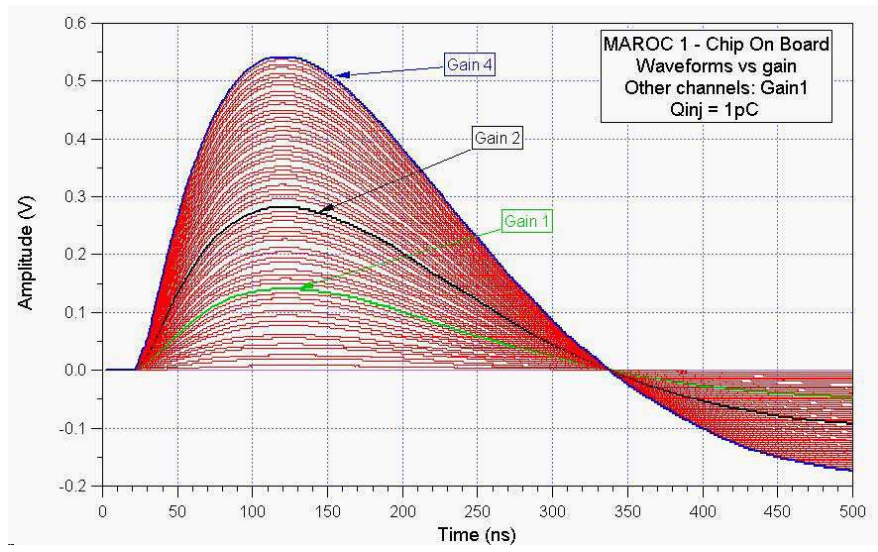

Fig. 8. Slow shaper waveforms taken with a fixed injection charge of $1 \mathrm{pC}$ and for variable preamplifier gains.

The linearity can be obtained from this plot by looking at the maximal amplitude of each waveform as a function of the gain, represented on fig. 9. The linear fit gives in this case residuals in the range of $[-1,1] \%$ in agreement with the requirements specified in the previous section.

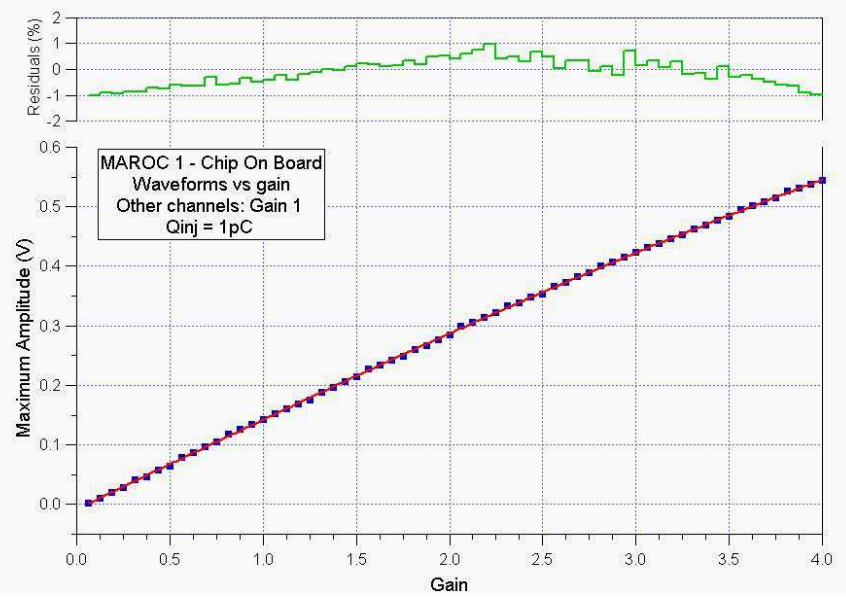

Fig. 9. Fit of the maximal amplitude of waveforms as a function of the preamplifier gain. The residuals of the fit are represented on top of the plot.

Similar measurements were performed with a fixed gain $(\mathrm{G}=1)$ and a variable injection charge $(0-5 \mathrm{pC})$. The linearity obtained was of $\pm 2 \%$ in most of the charge range, still in agreement with the requirements.

\section{E. $D A C$}

The linearity was checked by scanning the thermometer and mirror DACs and measuring the signal for each combination. Fig. 10 represents the evolution of the signal amplitude (Vdac) as a function of the thermometer-mirror combination, the thermometer varying from 0 to 10 and the mirror from 0 to 63 . By fitting this line in the region without saturation, we obtained a reasonable linearity of $\pm 2 \%$ on a large scale. 


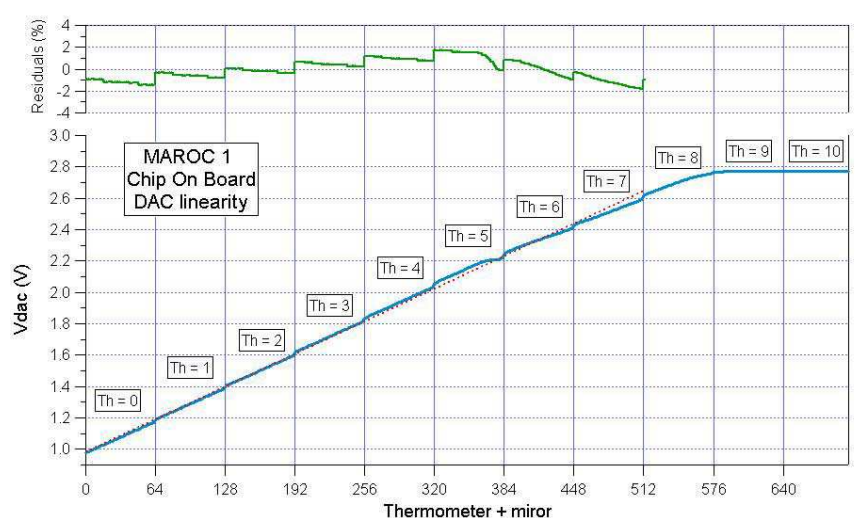

Fig. 10. DAC voltage as a function of the threshold set via the thermometer and mirror.

\section{F. Trigger output}

Well known S-curves were also studied. They correspond to the measurement of the trigger efficiency during a scan of the input charge while the threshold and the preamplifier gain are kept constant.

Fig. 11 represents the trigger efficiency as a function of the input charge for the 64 channels of a single chip. All channels were set at gain 1 and the threshold was fixed at $\mathrm{Vdac}=2.050 \mathrm{~V}$. We obtained $100 \%$ trigger efficiency for an input charge of approximately $50 \mathrm{fC}$ which corresponds to $1 / 3$ pe- as requested. The noise is equal to $1 \mathrm{fC}$ in agreement with the specifications.

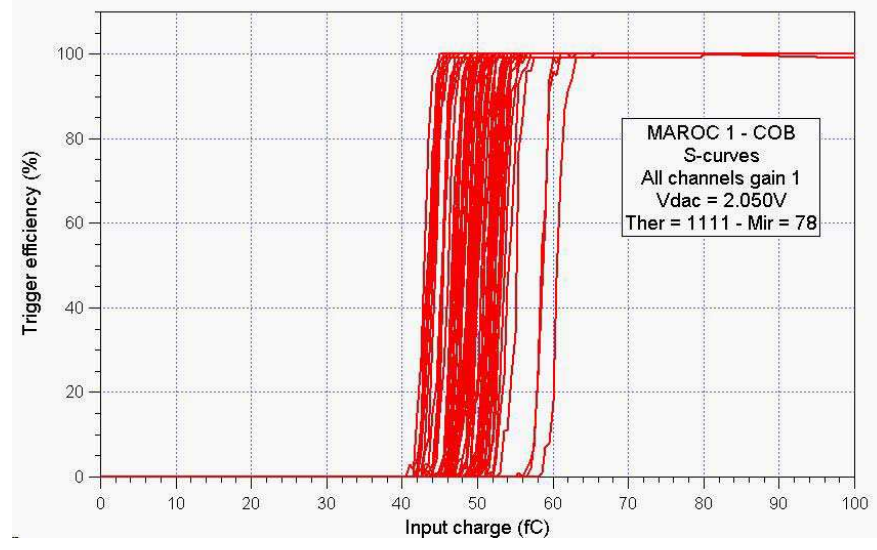

Fig. 11. Trigger efficiency as a function of the input charge, with a fixed threshold, for the 64 channels of a MAROC mounted on board; also called Scurves.

Fig. 12 represents the evolution of the 50\% trigger efficiency input charge as a function of the channel number. The mean value and rms are respectively 49.3 and $3.8 \mathrm{fC}$ leading to a reasonable dispersion of $7 \%$, taking into account that no gain tuning was performed to reduce it.

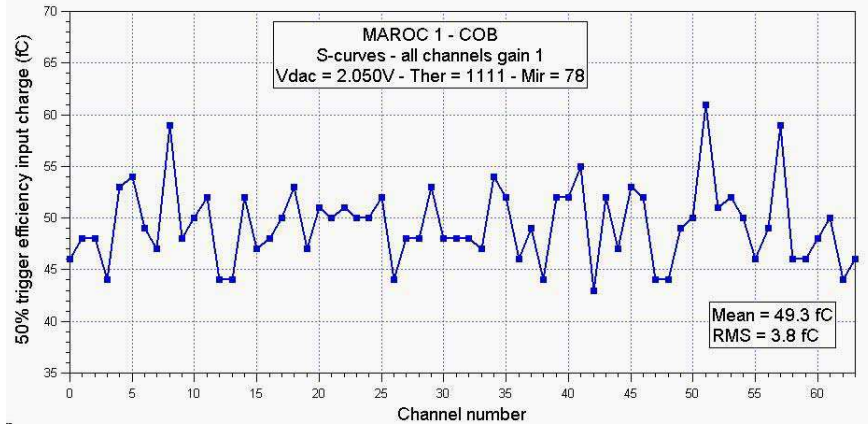

Fig. $12.50 \%$ trigger efficiency input charge versus channel number. The mean and rms are given.

Fig. 13 gives S-curve examples for different preamplifier gains (from 0.5 to 4 ). At high gain, the $100 \%$ trigger efficiency is reached at $15 \mathrm{fC}$ which corresponds to $1 / 10$ of photoelectron for a MAPMT gain of $10^{6}$.

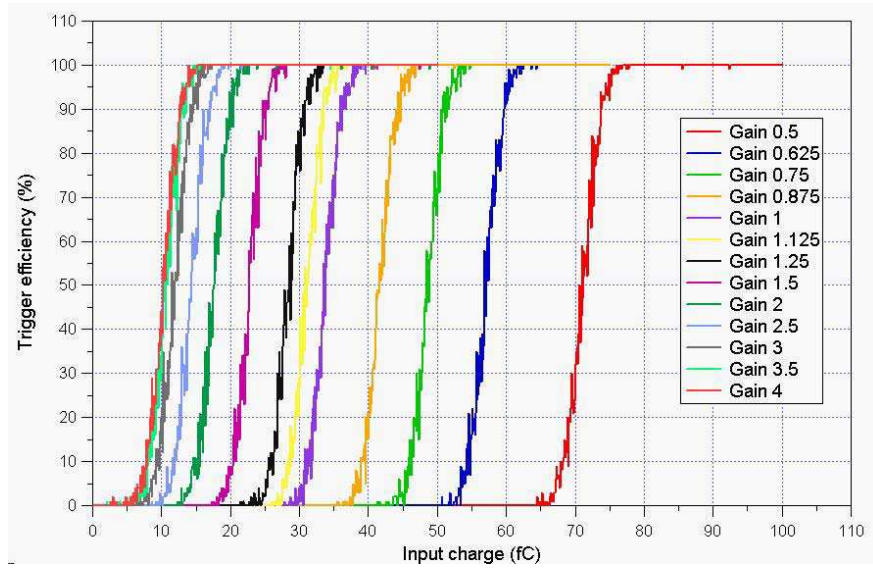

Fig. 13. S-curves for a single channel set at different preamplifier gains.

S-curves were also measured for all channels with different threshold values in the range $[1.85,2.13] \mathrm{V}$. The preamplifier gain was set to 1 for all measurements. Fig. 14 represents the $50 \%$ trigger efficiency input charge as a function of the channel number for 8 threshold values. The dispersion stays constant $(\sim 7-8 \%)$ while the threshold increases.

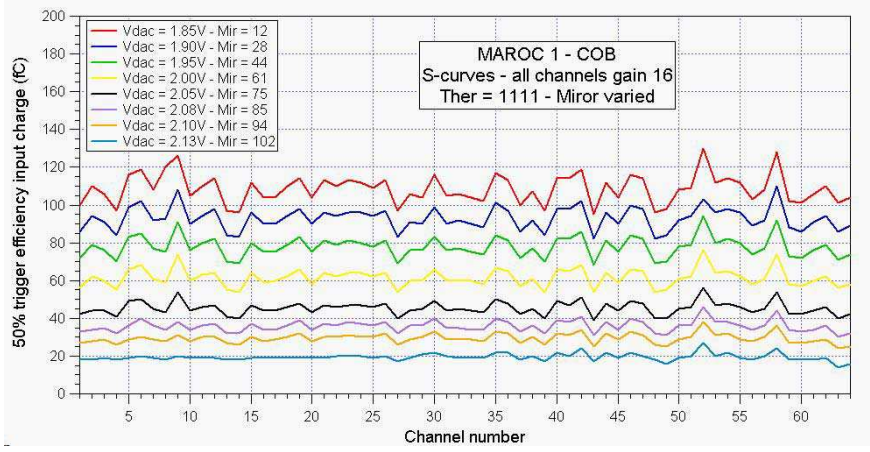

Fig. 14. 50\% trigger efficiency input charge versus channel number, for different threshold values. 


\section{G. Cross talk}

In order to study the cross talk, variable signal was sent to a central channel (34 in our example) and output trigger from this channel and the two neighboring (33 and 35) was recorded. The threshold was set in order to get $100 \%$ trigger efficiency at $50 \mathrm{fC}$ for the central channel. The maximal signal delivered was $20 \mathrm{pC}$ and the gain was 1 for all channels.

Fig. 15 represents the s-curves obtained for the three channels, with a large input charge range $([0-20] \mathrm{pC})$. No trigger signals were seen before $7.5 \mathrm{pC}$ for the two neighboring channels leading to a cross talk smaller than $1 \%$ as requested.

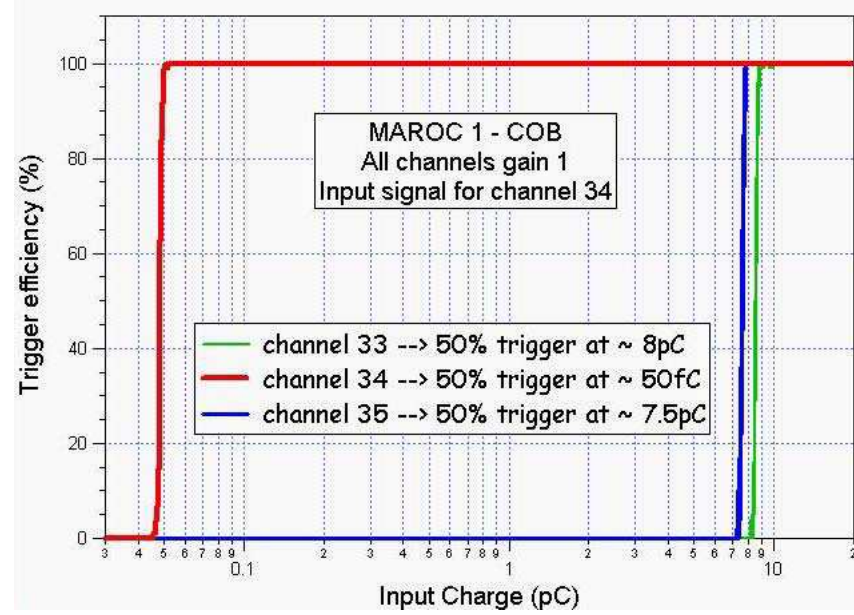

Fig. 15. S-curves for channel 34 and its two direct neighbours, up to an input charge of $10 \mathrm{pC}$

\section{H. Gain correction}

In preparation for the October 2006 test beam, some laboratory tests were carried out at CERN. The set-up consisted in a blue LED producing a light of 1-2 photoelectrons, a MAPMT and the complete electronic chain used during beam tests. Fig. 16 gives a view of the PMF (PM front-end) sandwich with in particular the MAROC mounted directly on board.
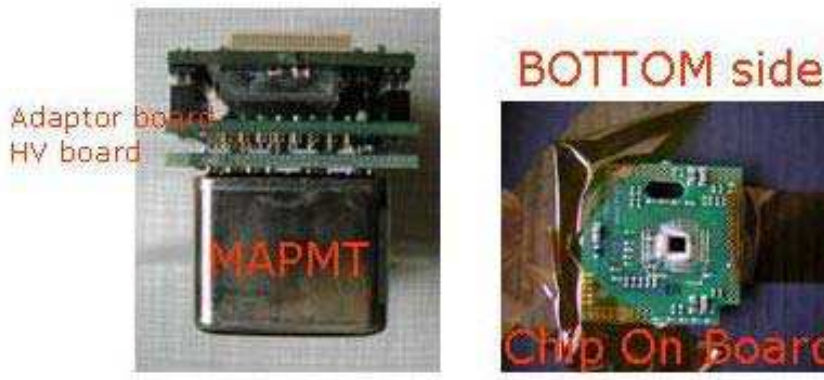

Fig. 16. Photomultiplier front-end electronic used during test beam and laboratory tests at CERN. It consists in a MAPMT, an HV board, an adaptor board and a board with the MAROC and the readout FPGA.
One of the main goals of these laboratory tests was to perform the gain equalization of the 5 MAPMTs used during test beam. This consists in tuning the gain channel by channel in order to equalize the response. Fig. 17 represents the relative counting rate versus the channel number for one MAPMT, before (blue diamonds) and after (pink squares) the gain equalization. Channels were set at gain 0.3 and in the gain range $0.125-1$ respectively before and after the tuning. The improvement of the homogeneity is quiet clear, the dispersion being reduced by a factor $3.6(22.5$ to $6.2 \%)$.

Gain equalization (PM \#4109)

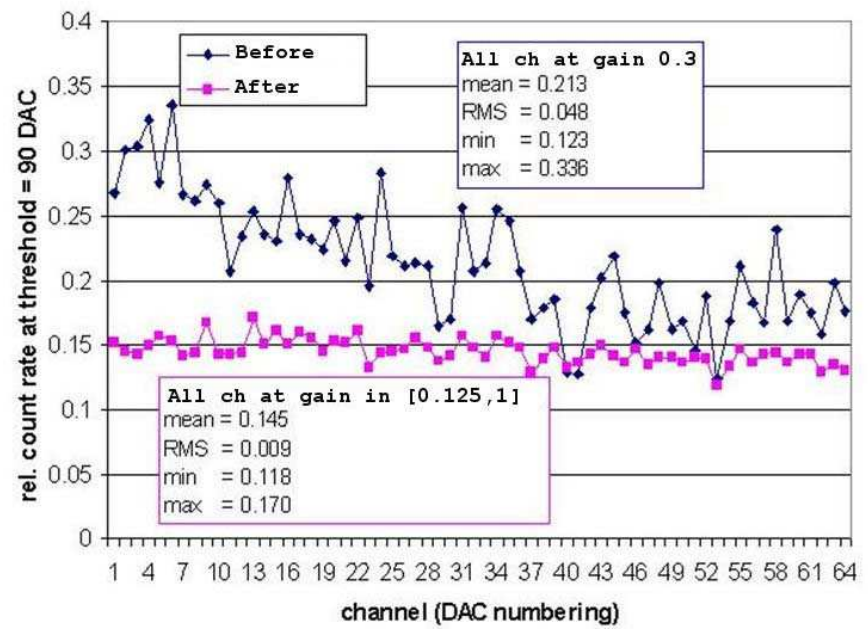

Fig. 17. Relative counting rate as a function of the channel number before (blue diamond) and after (pink square) gain correction.

\section{NEXT VERSION OF THE CHIP}

Despite the convincing results and performances obtained with the first version of MAROC, a new version has been designed in order to cure some effects like the substrate coupling. Moreover, MAROC won't be used only with the roman pots but also with LUCID ${ }^{1}$ (another ATLAS luminosity detector) and other experiments needing a MAPMT readout chip with different features. Thus the new version of the chip is integrating several additional features.

Fig. 18 gives a view of the layout of MAROC2 ${ }^{2}$ which has a substrate separation in order to remove the effect seen with the first version of the chip. A 12 bits Wilkinson ADC has been added to produce a multiplexed digital charge output. The fast shaper path has now two roads, the previously described bipolar fast shaper and the new unipolar fast shaper. Furthermore there are three discriminators instead of one for LUCID purpose. Finally, an $80 \mathrm{MHz}$ encoder has been added to produce an encoded version of the 64 trigger outputs.

\footnotetext{
${ }^{1}$ LUminosity measurement using Cerenkov Integrating Detector

${ }^{2}$ Name of the second version of MAROC.
} 


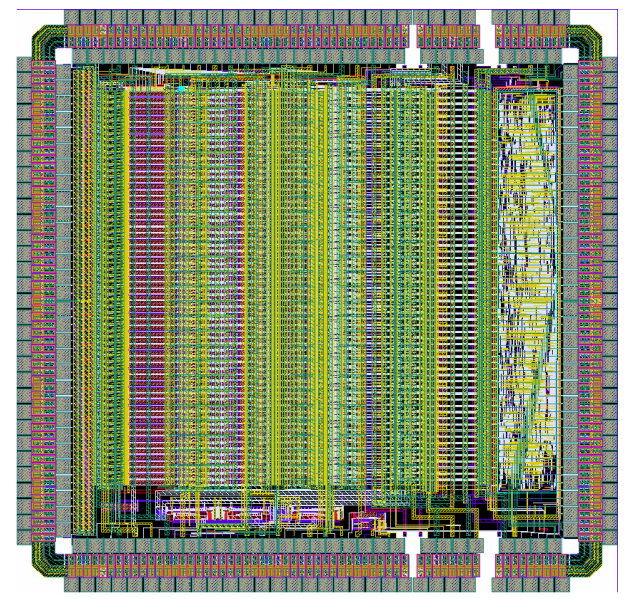

Fig. 18. Layout of the second version of MAROC

\section{CONCLUSION}

All the tests performed have showed a satisfying behavior of the chip. The substrate coupling effect, due to a large ground inductance, was solved by using a chip mounted directly on board up to a gain $\mathrm{G}=2$. The main requirements were fulfilled: $100 \%$ trigger efficiency at $1 / 3$ photoelectron, linearity better than $2 \%$, cross talk less than $1 \%$ and noise of $1 \mathrm{fC}$. The variable preamplifier gain was proved efficient by the tests carried out prior to the beam tests at CERN in October 2006. Finally MAROC was used successfully during these measurements with a prototype of the detector.

The new version of MAROC has been developed at LAL thanks to most of these results. It has some new features added in order to fit some new requirements and has substrate separation. It has been submitted in March 2006 and received in September 2006. It is presently tested at LAL and shows convincing performances.

\section{ACKNOWLEDGEMENT}

We would like to thank all the people who contributed directly or indirectly to this work. Especially the technical staff from LAL who produced and cabled the test boards and the one from CERN who supplied us the chips to be mounted on board.

\section{REFERENCES}

[1] ATLAS Collaboration, ATLAS Forward Detectors for Luminosity Measurement and Monitoring Letter of Intent, CERN/LHCC/2004-010.

[2] A. Lucotte et al., A front-end read out chip for the OPERA scintillating tracker. Nucl. Instr. And Meth. A521 (2004) 378-392.

[3] Hamamatsu web site, PM H7546B datasheet.

[4] S. Ask et al., Luminosity Measurement at ATLAS with a Scintillating Fiber Tracker, proceeding of IEEE 2006.

[5] LabVIEW web page: http://www.ni.com/labview/

[6] Igor Pro, web site: http://www.wavemetrics.com/

[7] Paw software web site: http://paw.web.cern.ch/paw/ 